\title{
La rosácea ocular en la frontera de la dermatología y la oftalmología
}

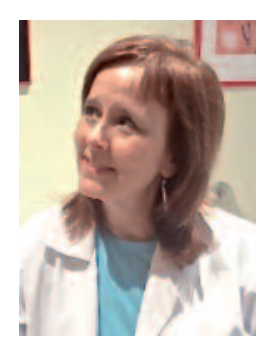

Carmen Fernández Jacob Unidad de Córnea y Segmento Anterior.

Hospital Universitario La Paz. Madrid.

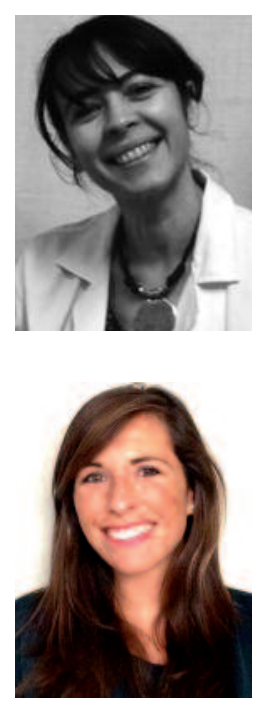

Ana Boto de los Bueis Unidad de Córnea y Segmento Anterior.

Hospital Universitario La Paz. Madrid.

\section{Fátima Sánchez-Carnerero}

\section{Casas}

Unidad de Córnea y Segmento Anterior.

Hospital Universitario La Paz. Madrid.

\section{DEFINICIÓN}

La rosácea es una enfermedad cutánea caracterizada por un eritema facial que se localiza en la nariz, las mejillas, la frente y el mentón, y que, con el tiempo, se hace persistente, acompañado de pápulas y pústulas ${ }^{1}$. En algunas ocasiones, se produce hiperplasia de tejidos blandos. Es una enfermedad muy común. Es más frecuente en personas de piel clara, durante la juventud y la madurez. Se observa un predominio del sexo femenino, aunque el desarrollo de hiperplasia de tejidos blandos de la nariz o rinofima es más común en hombres.

\section{ETIOPATOGENIA}

La etiología se desconoce, pero se han identificado varios factores que contribuyen a su desarrollo como: predisposición genética, trastornos psicológicos, parasitación por el ácaro Demodex folliculorum y alteraciones digestivas inespecíficas o relacionadas con Helicobacter pylori. Ninguno de ellos se ha confirmado de forma definitiva. Los pacientes presentan labilidad vasomotora o hiperreactividad vascular facial con tendencia a la vasodilatación ante varios estímulos como el calor, el frío, las radiaciones solares, las bebidas alcohólicas, las comidas calientes o las emociones. Son frecuentes los episodios de rubor.

\section{INTRODUCCIÓN: IMPORTANCIA DE LA COLABORACIÓN MULTIDISCIPLINARIA}

La rosácea es una enfermedad multidisciplinaria, que obliga, en muchas ocasiones, a la colaboración entre dos especialidades que tienen muchas afinidades entre sí, como son la dermatología y la oftalmología. 
El término rosácea ocular se utiliza para designar un espectro de hallazgos oculares con telangiectasias en el borde palpebral, con frecuencia, sin afectación significativa del resto de la cara.

En los casos en los que debuta con los síntomas oftalmológicos, que, en principio, son algo inespecíficos, y también porque, a veces, la enfermedad se presenta con una morbilidad ocular significativa y una afectación cutánea relativamente leve, es muy necesario un diagnóstico preciso desde el punto de vista dermatológico, que ayudará a poder tratar mejor la enfermedad ocular.

\section{EPIDEMIOLOGÍA}

\section{DE LA ENFERMEDAD OCULAR}

Es un trastorno crónico que afecta más a las mujeres que a los hombres y que suele iniciarse entre los 25 y los 50 años de edad. Esta dermatosis aparece, sobre todo, en la zona de la frente, la nariz, las mejillas y la barbilla.

Posee un componente vascular (eritema y telangiectasia) con o sin componente acneiforme (pápulas, pústulas y nódulos). No se observan comedones $^{2,3}$.

Entre el 6 y el $18 \%$ de los pacientes con rosácea desarrollan complicaciones oculares.

Es ciertamente una enfermedad más frecuente de lo que se cree, que merece atención no solo por su morbilidad, sino también por sus secuelas ${ }^{4}$.

\section{RELACIONES ETIOLÓGICAS ENTRE LA ENFERMEDAD DERMATOLÓGICA Y LA OCULAR}

Se desconoce la causa de la rosácea y también la relación entre la enfermedad cutánea y la ocular.

Aunque no existe evidencia científica de alto nivel de que la enfermedad cutánea se relacione con la presencia de microorganismos estafilocócicos positivos al manitol, estos microorganismos están presentes en los párpados y en la conjuntiva de la mayoría de los casos que responden al tratamiento con tetraciclinas ${ }^{5}$.

\section{MANIFESTACIONES OCULARES DE LA ROSÁCEA}

\section{Patología palpebral}

\section{Blefaritis y meibomitis}

El término blefaritis abarca un grupo de enfermedades frecuentes que afectan a la base de las pestañas (blefaritis anterior) y a las glándulas de Meibomio (blefaritis posterior) ${ }^{4}$.

La blefaritis anterior se caracteriza por la acumulación de material escamoso alrededor de la base de las pestañas y eritema del borde anterior del párpado. Existen dos variedades: estafilocócica y seborreica. La sintomatología es muy inespecífica ${ }^{6}$.

La meibomitis se debe a una secreción excesiva de las glándulas de Meibomio que cursa con eritema del margen palpebral y condensación de secreciones en los orificios de las glándulas (fig. 1). Se acompaña de quemazón, lagrimeo, prurito, sequedad, irritación y fotofobia. Es muy importante saber que dos tercios de los pacientes con esta sintomatología tienen una rosácea y que esta enfermedad dermatológica puede ocasionar eritema, telangiectasias, pápulas, pústulas foliculares e hipertrofia de las glándulas sebáceas, que habrá que valorar en cada caso.

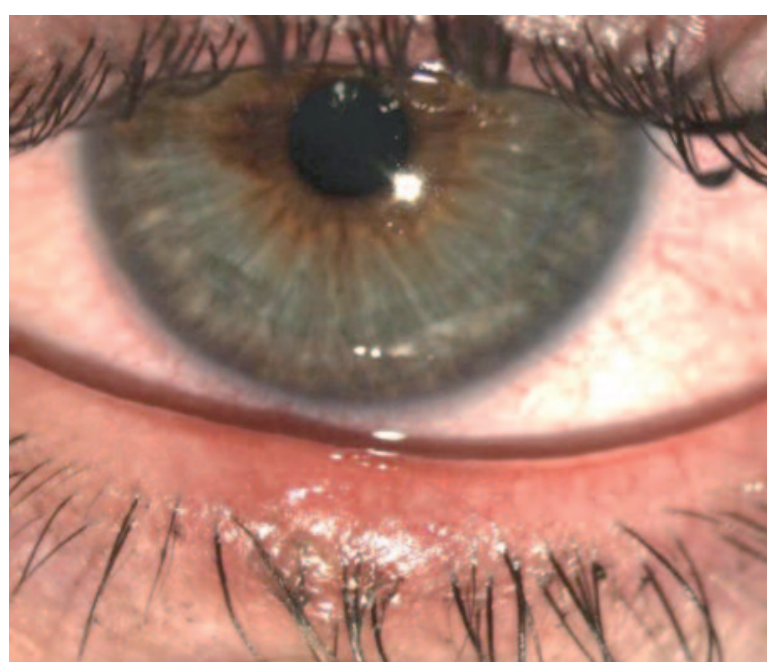

Figura 1. Chalación secundario a blefaritis posterior. 


\section{Chalación}

El chalación es la inflamación nodular estéril y crónica de unas glándulas de Zeis o de Meibomio. Es una reacción granulomatosa frente al material sebáceo que ha sido expulsado de una glándula taponada al tejido que la circunda y que acompaña, a veces, a la rosácea como patología palpebral.

\section{Patología conjuntival}

\section{Telangiectasias conjuntivales}

Los vasos sanguíneos de la conjuntiva pueden dilatarse como los de la piel, los párpados, la nariz y el rostro.

Estos vasos dilatados son más frecuentes en las áreas interpalpebrales de la conjuntiva bulbar, y pueden ser grandes y tortuosos y llevar, a veces, a un diagnóstico erróneo de conjuntivitis infecciosa ${ }^{7}$.

\section{Conjuntivitis nodular}

Son pequeñas elevaciones de la conjuntiva bulbar grises y muy vascularizadas, localizadas casi siempre próximas al limbo en la zona interpalpebral, semejantes a una flicténula (fig. 2).

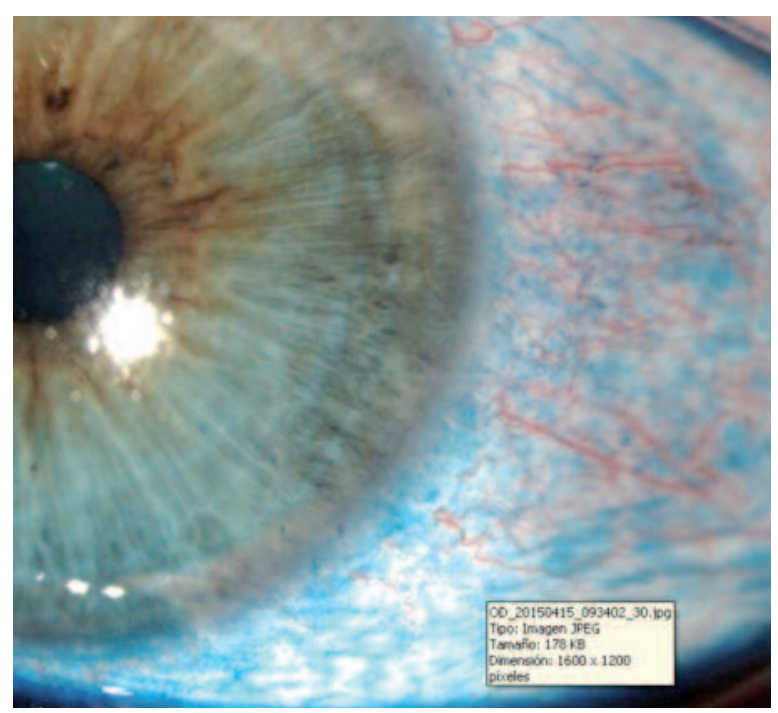

Figura 2. Tinción con verde de lisamina de los vasos conjuntivales.

\section{Patología corneal}

\section{Queratitis rosácea}

La córnea puede verse afectada ampliamente con cicatrización y vascularización. La queratitis rosácea cursa con dolor, fotofobia y sensación de cuerpo extraño. En casos de rosácea ocular avanzados, se produce la sustitución del epitelio corneal por un epitelio conjuntival vascularizado, por un daño irreversible de las células madre del limbo esclerocorneal sometidas a una inflamación crónica.

\section{Queratopatía punteada superficial}

Afecta a los dos tercios inferiores de la córnea y acompaña, a menudo, a la blefaritis o la meibomitis.

\section{Vascularización periférica}

Es habitual que un trío de vasos superficiales crezcan sobre la córnea, por lo general, en el área interpalpebral inferior, pudiendo asociarse a infiltrados superficiales en el borde central.

\section{Queratitis marginal}

Afecta especialmente a la córnea inferonasal o inferotemporal periférica.

\section{Extensión circunferencial}

La extensión circunferencial de las úlceras marginales se produce, generalmente, cuando se tiende a una progresión con brotes intermitentes de la enfermedad. Las remisiones y exacerbaciones de la enfermedad oftálmica pueden producirse con independencia del curso de la enfermedad cutánea $^{6}$ (fig. 3).

\section{Adelgazamiento corneal}

Se produce en casos graves, pudiendo llegar a una perforación corneal por derretimiento del estroma corneal periférico cuando ha habido brotes graves repetidos y también cuando se han utilizado los corticoides tópicos de forma imprudente ${ }^{6}$ (fig. 4). 


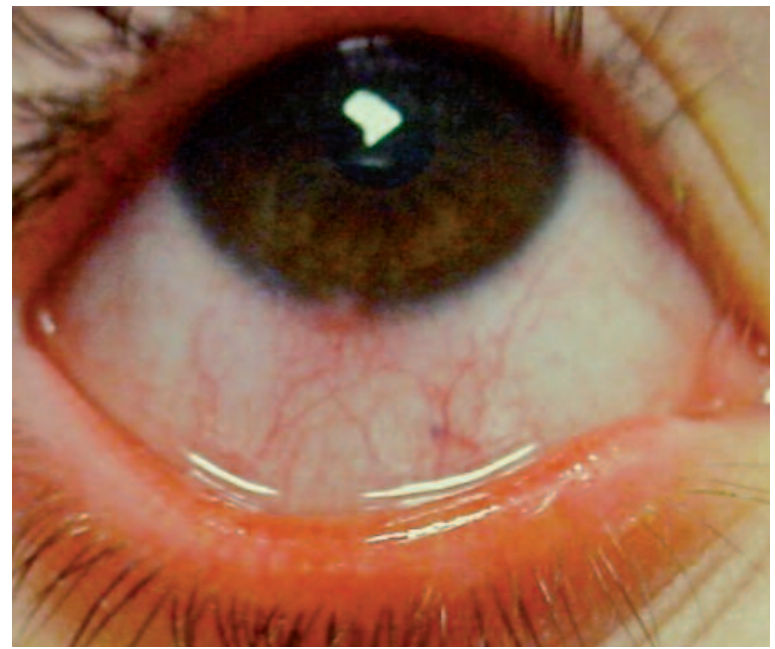

Figura 3. Flicténula conjuntival.

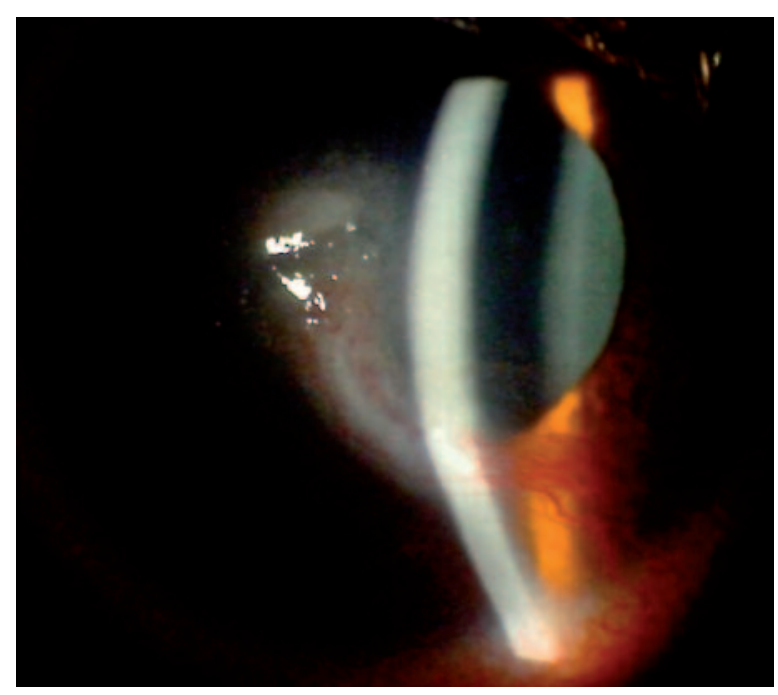

Figura 4. Flicténula corneal con neovasos estromales.

\section{TRATAMIENTO MÉDICO}

\section{Formas leves}

Las afectaciones palpebrales, conjuntivales y corneales leves pueden tratarse de forma tópica:

- Compresas calientes e higiene con masaje palpebral para la blefaritis posterior.

- Expresión de las glándulas de Meibomio tras aplicar calor local en la meibomitis (existen comercializadas gafas adaptadas para la aplicación de calor a los párpados).

- Hidratación de la superficie ocular con lágrimas artificiales y pomada de vitamina A.

- Pomada de ácido fusídico (dos veces al día) al acostarse durante cuatro semanas.

- Aceite del árbol del té al $50 \%$ en aplicación tópica en el borde de los párpados si se comprueba infestación por Demodex folliculorum ${ }^{7}$.

- Colirio de fluorometolona al 0,1\% como medida a corto plazo.

\section{Formas graves}

El paciente con rosácea grave debe estar bajo control conjunto de un dermatólogo y un oftalmólogo.

El tratamiento más eficaz es la tetraciclina sistémica, en dosis de $250 \mathrm{mg}$ cuatro veces al día. El efecto terapéutico de la tetraciclina no está relacionado con su acción antibacteriana; se desconoce por qué la rosácea responde a la tetraciclina $^{7}$.

La mejoría de la enfermedad ocular suele durar hasta seis meses después de dejar el tratamiento en las formas de afectación corneal recurrente.

Si no se tolera la tetraciclina, puede utilizarse una tetraciclina semisintética, la doxiciclina (100 mg al día $)^{8}$ o la ampicilina o la eritromicina (ambas en dosis de $250 \mathrm{mg}$ /día), o el metronidazol para impedir la recidiva de la enfermedad y sus posibles efectos devastadores sobre la córnea.

Hay que prestar especial atención al uso de los corticoides tópicos, que pueden ser muy útiles para reducir la inflamación ocular, la invasión vascular y la infiltración corneal, pero hay que asegurarse de que los infiltrados corneales no son infecciosos antes de iniciar este tratamiento, pues, entonces, están contraindicados, y también tener en cuenta siempre que, al ser una enfermedad crónica y precisar tratamientos prolongados, el uso de los corticoides puede favorecer el adelgazamiento y la perforación corneal ${ }^{9}$.

También puede ser útil el tratamiento con colirios de ciclosporina cada 12 horas o el tratamien- 
to con colirios de suero autólogo preparados con la sangre del paciente, que mejoran las erosiones corneales y el ojo seco asociado a la rosácea ocular. En los casos graves con lisis corneal y riesgo de perforación ocular, puede ser necesario el tratamiento con fármacos inmunosupresores o inmunomoduladores sistémicos ${ }^{10}$.

\section{TRATAMIENTO QUIRÚRGICO}

En casos muy graves, puede ser necesario realizar un injerto corneal cuando la córnea cicatriza extensamente, aunque los resultados a largo plazo no son satisfactorios ${ }^{11}$.

\section{BIBLIOGRAFÍA}

1. Guerra-Tapia A. Rosa, rosae. Jugando con las palabras. Más Dermatol. 2014;(23):25-7.

2. Awais M, Anwar MI, Iftikhar R, Iqbal Z, Shehzad N, Akbar B. Rosacea - the ophtalmic perspective. Cutan Ocul Toxicol. 2015;34(2):161-6.
3. Guerra Tapia A, González-Guerra E, Gallego Álvarez S. Acnés. Rosácea. En: Manual de dermatología y venereología. Atlas y texto. Madrid: Editorial Médica Panamericana; 2008. p. 713-31.

4. Yildirim Y, Olcucu O, Agca A, Karakucuk Y, Alagoz N, Mutaf $C$, et al. Topographic and biomechanical evaluation of corneas in patients with ocular rosacea. Cornea. 2015;34(3): 313-7.

5. Knight AG, Vickers CF. A follow-up of tetracycline-treated rosacea. With special reference to rosacea keratitis. $\mathrm{Br} J$ Dermatol. 1975;93(5):577-80.

6. Brown SI, Shahinian L Jr. Diagnosis and treatment of ocular rosacea. Ophalmology. 1978;85(8):779-86.

7. Wladis Ej. Intraductal meibomian gland probing in the management of ocular rosacea. Ophthal Plast Reconstr Surg. 2012; 28(6):416-8.

8. Gao YY, Di Pascuale MA, Elizondo A, Tseng SC. Clinical treatment of ocular demodecosis by lid scrub with tea tree oil. Cornea. 2007;26(2):136-43.

9. Schechter BA, Katz RS, Friedman LS. Efficacy of topical cyclosporine for the treatment of ocular rosacea. Adv Ther. 2009; 26(6):651-9.

10. Sobolewska B, Doycheva D, Deuter C, Pfeffer I, Schaller M, Zierhut M. Treatment of ocular rosacea with once-daily lowdose doxycycline. Cornea. 2014;33(3):257-60.

11. Hopkinson D, Moradi Tuchayi S, Alinia H, Feldman SR. Assessment of rosacea severity: A review of evaluation methods used in clinical trials. J Am Acad Dermatol. 2015;73(1):138-43.e4. 